\title{
LA FACTIBILIDAD DEL FINANCIAMIENTO EN MÉXICO
}

FINANCIAL FEASIBILITY IN MEXICO

\author{
Mtra. María Verónica Altagracia Lara Andrade ${ }^{\text {a }}$ \\ Mtro. Genaro Roberto López Aguilar ${ }^{\text {b }}$ \\ Dra. Patricia Vázquez Juárez ${ }^{\mathrm{c}}$ \\ Nohemí Botello Marín ${ }^{\text {d }}$
}

\author{
a Benemérita Universidad Autónoma de Puebla \\ Facultad de Contaduría Pública vlaraan@gmail.com \\ ${ }^{\mathrm{b}}$ Benemérita Universidad Autónoma de Puebla \\ Facultad de Ingeniería, grlopeza@gmail.com \\ ${ }^{\mathrm{c}}$ Benemérita Universidad Autónoma de Puebla \\ Facultad de Contaduría Pública, patriciavjuarez@yahoo.com.mx \\ d Benemérita Universidad Autónoma de Puebla \\ Facultad de Contaduría Pública, nohemibm97@gmail.com
}

\section{RESUMEN}

El financiamiento representa una importante herramienta de apalancamiento para los desafíos en la vida de las Micro Pequeñas y Medianas Empresas (MIPYME). Este trabajo tiene como objetivo principal mostrar que en México, han existido en los últimos años, importantes programas de financiamiento por parte instituciones gubernamentales y no gubernamentales, sin embargo, hay obstáculos para su óptimo aprovechamiento. La metodología usada tiene un enfoque mixto, elaborando primero un análisis documental, exploratorio sobre conceptos relacionados con la importancia del financiamiento para las MIPYME, en libros, revistas y documentos de investigación. En la parte cualitativa se hizo un estudio fenomenológico, analizando bases de datos entre las que se encuentran, los resultados de encuestas realizadas a las empresas por el Instituto Nacional de Estadística y Geografía (INEGI), en particular la Encuesta Nacional sobre Productividad y Competitividad de las Micro, Pequeñas y Medianas Empresas en 2015 (ENAPROCE), así como los reportes emitidos por La Organización para la Cooperación y el Desarrollo Económico (OCDE, 2018), el Instituto Nacional del Emprendedor (INADEM, 2018) y la Sociedad Financiera de Objeto Múltiple (KONFIO, 2018). Además de los diferentes portales de bancos comerciales que ofertan financiamiento, comparando sus costos y requisitos. Procediendo a sintetizar los resultados mediante tablas y gráficos. Entre los resultados se comprueba que hay necesidad de financiamientos adecuados, de fácil acceso, con intereses bajos, sin intermediarios, así como, una mayor difusión de los apoyos existentes y capacitación constante, que permita a las empresas crecer y administrar sus recursos financieros de forma más eficiente. Una de las limitaciones más 
importantes enfrentadas, es el que los datos analizados observan información solo hasta el año 2018 por lo que se contemplan únicamente programas y políticas de gobiernos anteriores. Es interesante seguir investigando sobre este tema, dadas las expectativas de mejora y cambios generadas en este nuevo periodo de gobierno.

PALABRAS CLAVE: Microempresas; Financiamiento; Costo.

\section{ABSTRACT}

Financing represents an important leverage tool for the challenges in the life of Micro and Small Businesses (MSMEs). The main objective of this work is to show that in Mexico, important financing programs have been in place in recent years by governmental and non-governmental institutions. The methodology used has a mix approach, the elaboration of a documentary analysis, the exploratory of concepts related to the importance of financing for MSMEs, in books, journals and research documents. In the qualitative part a phenomenological study was made, analyzing the bases of the data that are found, the results of the surveys conducted by the National Institute of Statistics and Geography (INEGI), in particular the National Survey on Productivity and Competitiveness of Micro, Small and Medium Enterprises in 2015 (ENAPROCE), as well as the reports issued by the Organization for Economic Cooperation and Development (OECD 2018), the National Institute of the Entrepreneur (INADEM, 2018) and the Multiple Purpose Financial Society ( KONFIO 2018). In addition to the different portals of commercial banks that offer financing, comparing their costs and requirements. Proceeding to synthesize the results through tables and graphs. The results show that there is a need for financial means, easily accessible, with low interests, without intermediaries, as well as, with greater diffusion of existing supports and constant training, so that companies grow and adapt to their financial resources. One of the most important limitations is found in the analyzed data. Only until 2018. It is interesting to continue investigating on this subject, given the expectations of improvement and changes in this new period of government.

KEYWORDS: Micro companies; Financing; Cost.

\section{INTRODUCCIÓN}

El financiamiento es una herramienta importante en el desarrollo y supervivencia de las MIPYMES. En nuestro país han existido en los últimos años algunos programas de financiamiento y apoyo a las empresas que ofertan diversas instituciones de crédito e instituciones gubernamentales.

Muchas veces no se tiene acceso a un tipo de financiamiento debido a que se desconoce el procedimiento para obtenerlo, es difícil conseguirlo por los excesivos tramites, o no es entregados de manera oportuna, otras veces aunque se obtiene el recurso financiero este llega a ser usado para un objetivo diferente al que se planteó en un inicio, o en otros casos llega a ser tan costoso que resulta desfavorable para la

Lara, M. V. A., López Aguilar, G. R., Vázquez Juárez, P. \& Botello, N. 
empresa.

\section{PLANTEAMIENTO}

Si bien es cierto que el costo de financiamiento en México se encuentra entre los más altos a nivel mundial, también se puede mencionar a los diversos programas de apoyo que existen como los que ofrece NAFIN, FIRA, BANCOMEXT, INADEN, FONDO PYMES, entre otros, que pueden ser una buena opción.

Una vez obtenido el financiamiento la empresa debe cumplir con el compromiso de pagarlo sin caer en la morosidad, es decir, la empresa debe ser productiva para que pueda crecer. Las MIPYMES representan el $97.6 \%$ de las empresas del país y dan empleo al $75 \%$ del personal ocupado (INEGI, 2016), por lo que se les debe poner atención especial para su desarrollo y crecimiento, en beneficio de nuestro país.

Pregunta de investigación: ¿Puede una Micro pequeña y mediana empresa (MIPYME) en México obtener un financiamiento adecuado a su empresa y cumplir con el compromiso de pagarlo?

\section{OBJETIVOS}

Mostrar que en México aunque si bien existen apoyos y financiamiento por parte de instituciones de crédito, así como por parte de los gobiernos federal y estatal para las MIPYMES, estos, no siempre son fáciles de obtener, no se conoce sobre su existencia, e inclusive pueden parecer no favorables para la empresa.

Mostrar como algunos de los requisitos generales para obtener un financiamiento pueden llegar a convertirse en arduos procesos, algunas veces con pagos altos por comisiones que llegan a incrementar de manera importante el costo total del financiamiento.

Presentar tablas y gráficos que permitan de manera estructurada comparar los diferentes valores de las variables de estudio, como lo son número de empresas, tamaño de crédito, porcentaje de solicitudes para los diferentes rangos entre otras.

\section{MARCO TEÓRICO CONCEPTUAL}

Esta investigación inicia con la definición de Microempresa, financiamiento y posteriormente se analizarán a las MIPYMES en cuanto al financiamiento.

\section{IV.I Microempresa (MIPYMES)}

La Secretaría de Economía, clasifica a las empresas como a continuación se presenta el siguiente cuadro: 
Tabla 1.

Clasificación de las PYMES

\begin{tabular}{lccccc}
\hline \multicolumn{1}{c}{ CLASIFICACION DE LAS PYMES } & & \\
\hline PYMES & Trabajadores & $\begin{array}{c}\text { Ventas (millones de } \\
\text { pesos) }\end{array}$ & $\begin{array}{c}\text { Total } \\
\text { empresas }\end{array}$ & $\begin{array}{c}\text { Empleo en el } \\
\text { país }\end{array}$ & PIB \\
\hline Microempresa & 1 - 10 & 4 & $95 \%$ & $40 \%$ & $15 \%$ \\
\hline Pequeñas & 11 a 30 & 4 a 100 & $3 \%$ & $15 \%$ & $14 \%$ \\
\hline Medianas & 31 a 100 & 100 a 250 & $1 \%$ & $17 \%$ & $22 \%$ \\
\hline
\end{tabular}

Fuente: Elaboración propia con datos del Portal Web Secretaría de Economía (2018)

Las microempresas son todos aquellos negocios que tienen menos de 10 trabajadores, generan anualmente ventas hasta por 4 millones de pesos y representan el 95 por ciento del total de las empresas y el 40 por ciento del empleo en el país; además, producen el 15 por ciento del Producto Interno Bruto (Secretaría de Economía, 2018).

\section{IV.II Definición de financiamiento.}

De acuerdo con la Real Academia se define al financiamiento como: "la acción de financiar", y "financiar es aportar el dinero necesario para el funcionamiento de una empresa". También se define como "sufragar los gastos de una actividad, de una obra, etc." (RAE, 2019).

\section{IV.II.I Fuentes de financiamiento (Rodríguez et al., 2014):}

a) Financiamiento interno (autofinanciamiento): reservas o autofinanciación por enriquecimiento (beneficios retenidos) amortizaciones, provisiones y previsiones (autofinanciación por mantenimiento). El financiamiento interno es una fuente de financiación que disponen sólo aquellas empresas que ya se encuentran operando en el mercado y proviene fundamentalmente del excedente de las operaciones.

b) Financiamiento externo: fondos prestables de diversas fuentes, que son tomados o generados en el mercado de dinero y/o capitales. En general la financiación de la inversión inicial de un proyecto es a mediano o largo plazo. Por tal razón, las alternativas de financiación son las siguientes:

- Préstamos y/o créditos bancarios (ver tabla no.2)

- Emisión de acciones ordinarias o comunes

- Emisión de acciones preferentes o de inversión

- Emisión de bonos

- Arrendamiento financiero.- Créditos destinados a financiar los activos de la empresa.

- Productos derivados (opciones, futuros forwards, swaps) 
Por lo que se refiere a los créditos bancarios a continuación se presenta el siguiente cuadro:

Tabla 2.

Créditos bancarios

\begin{tabular}{|c|c|c|}
\hline \multicolumn{3}{|c|}{ CRÉDITOS BANCARIOS } \\
\hline \multirow{9}{*}{$\begin{array}{c}\text { A corto } \\
\text { plazo }\end{array}$} & Quirografarios & Contrato de mutuo interés \\
\hline & Directo con garantía colateral & Un pagaré que especifica la garantía \\
\hline & Prendario & $\begin{array}{l}\text { Equivale a un \% del valor comercial de las mercancías } \\
\text { o valores }\end{array}$ \\
\hline & Descuentos Mercantiles & $\begin{array}{l}\text { Recuperación inmediata de los fondos invertidos en } \\
\text { documentos por cobrar. Ejemplo: Factoraje } \\
\text { financiero }\end{array}$ \\
\hline & Simple & Prestamos avalados por pagarés, en una sola entrega \\
\hline & Revolvente & Disposiciones hasta que la línea lo permita \\
\hline & Comerciales & Facilitan el comercio interior o exterior \\
\hline & Tarjeta de crédito & $\begin{array}{l}\text { Disposiciones por varios canales (banca electrónica, } \\
\text { establecimientos, etc.) }\end{array}$ \\
\hline & Aceptaciones bancarias & $\begin{array}{l}\text { Letras de cambio emitidas por empresas a su propia } \\
\text { orden y aceptadas por sociedades nacionales de } \\
\text { crédito }\end{array}$ \\
\hline $\begin{array}{l}\text { A mediano } \\
\text { plazo }\end{array}$ & Habilitación o avío & $\begin{array}{l}\text { Habilita a la empresa de sus gastos directos de } \\
\text { fabricación, abastece al negocio de materias primas y } \\
\text { materiales }\end{array}$ \\
\hline \multirow{3}{*}{$\begin{array}{l}\text { A largo } \\
\text { Plazo }\end{array}$} & Refaccionario & Destinados para la adquisición de activos fijos \\
\hline & Con garantía Hipotecaria & La garantía es sobre bienes inmuebles \\
\hline & Simple con o sin garantía & $\begin{array}{l}\text { Contrato dirigido a proyectos y ampliaciones de gran } \\
\text { tamaño }\end{array}$ \\
\hline
\end{tabular}

Fuente: Elaboración propia con base en Herrera (1998)

Muchos de estos créditos los ofrece la Banca de Desarrollo por medio de la Banca Comercial y los Intermediarios Financieros no bancarios (Sofomes, Uniones de Crédito, entre otros). También hay programas del gobierno como Fondo Pymes, INADEM, Red entre otros. A continuación se presenta una breve descripción sobre las Instituciones Financieras de la Banca de Desarrollo:

\section{IV.III Nacional Financiera (NAFIN)}

Contribuye al desarrollo económico de México, facilitando el acceso de las MIPYMES, emprendedores y proyectos de inversión prioritarios al financiamiento y otros servicios de desarrollo empresarial, así como contribuir a la formación de mercados financieros y fungir como fiduciario y agente financiero del Gobierno Federal, que permita impulsar la innovación, mejorar la productividad, la competitividad, la generación de empleos y el 
crecimiento regional (Nafin, 2008). En el siguiente cuadro se muestran los tipos de financiamiento de los Fondos NAFIN.

Tabla 3.

Financiamiento empresarial fondos NAFIN

FINANCIAMIENTO EMPRESARIAL FONDOS NAFIN

\begin{tabular}{|c|c|c|}
\hline \multicolumn{2}{|c|}{ Financiamiento Empresarial } & $\begin{array}{l}\text { Crédito para comprar insumos; remplazar, modernizar o } \\
\text { adquirir algún mobiliario; o invertir en adecuaciones y } \\
\text { mejoras al local. }\end{array}$ \\
\hline $\begin{array}{l}\text { Financiamiento } \\
\text { CSOLAR }\end{array}$ & $14.50 \%$ & $\begin{array}{l}\text { Financiamiento para que generes energía eléctrica solar en } \\
\text { tu negocio. }\end{array}$ \\
\hline Ven a Comer & varia & $\begin{array}{l}\text { Financiamiento dirigido a restaurantes y empresas que } \\
\text { brinden servicios de preparación de alimentos para } \\
\text { banquetes }\end{array}$ \\
\hline Impulso Económico & $14.50 \%$ & $\begin{array}{l}\text { Nafin a través de la red de intermediarios financieros } \\
\text { incorporados al programa de Financiamiento Empresarial } \\
\text { ofrece condiciones favorables de acceso al financiamiento a } \\
\text { las pymes mexicanas. }\end{array}$ \\
\hline $\begin{array}{l}\text { Estímulo al Buen } \\
\text { Contribuyente }\end{array}$ & TIIE +9p & $\begin{array}{l}\text { Crédito que reconoce a las empresas formales que son } \\
\text { contribuyentes cumplidos, }\end{array}$ \\
\hline Textil, Vestido y Moda & TIIE+8 P & $\begin{array}{l}\text { Financiamiento para impulsar la modernización, innovación } \\
\text { y desarrollo de las empresas de la industria textil, del } \\
\text { vestido y de la moda. }\end{array}$ \\
\hline $\begin{array}{l}\text { Mejora Sustentable } \\
\text { en Vivienda }\end{array}$ & $20 \%$ & $\begin{array}{l}\text { Crédito para la instalación de equipos que permitan el } \\
\text { aprovechamiento de energía solar }\end{array}$ \\
\hline Taxi Estrena & $14.50 \%$ & Hibrido y convencional \\
\hline Ecocréditos & $20 \%$ & $\begin{array}{l}\text { Crédito para la sustitución de equipos obsoletos por unos } \\
\text { nuevos de tecnología de punta }\end{array}$ \\
\hline Cuero y Calzado & TIIE $+8 p$ & $\begin{array}{l}\text { Crédito en condiciones competitivas para capital de trabajo } \\
\text { y activos fijos }\end{array}$ \\
\hline Empresas Gasolineras & $\mathrm{TIEE}+8 p$ & $\begin{array}{l}\text { Crédito para mejorar tu estación de servicio y/o integrarla } \\
\text { con servicios adicionales }\end{array}$ \\
\hline Radiodifusoras & & Financiamiento dirigido a empresas de radiodifusión \\
\hline
\end{tabular}

Fuente: Elaboración propia con datos del portal web de Nafinsa (2018).

\section{IV.IV Fideicomisos Instituidos en Relación con la Agricultura (FIRA)}

Es una Institución dedicada a apoyar el desarrollo de los sectores rural, agropecuario, forestal y pesquero del país a través de intermediarios financieros y empresas especializadas, otorgamos crédito, garantías, capacitación, asistencia técnica y transferencia de tecnología para que productores y empresas rurales para que, puedan iniciar o hacer crecer sus proyectos productivos. Son cuatro fideicomisos públicos que tienen el carácter de entidades de la Administración Pública Federal, en los que funge como fideicomitente la Secretaría de Hacienda y Crédito Público y como fiduciario el 
Banco de México (FIRA, 2018).

Los créditos otorgados por FIRA a corto plazo son: Crédito por conceptos de avío y capital de trabajo, crédito prendario, arrendamiento, reporto, préstamo quirografario, crédito de liquidez. Y a largo plazo: Crédito refaccionario y Factoraje. Adicionalmente a lo anterior, se otorgan créditos para actividades no tradicionales del sector rural: Financiamiento rural y Microcrédito.

\section{IV.V Banco Nacional de Comercio Exterior (Bancomext).}

Es el banco de desarrollo que apoya al comercio exterior en México, y su objetivo es promover el acceso de las pequeñas y medianas empresas al financiamiento para elevar el contenido nacional en sus productos de exportación.

Tabla 4.

Intermediarios Financieros BANCOMEXT

\begin{tabular}{ll}
\hline \multicolumn{2}{c}{ INTERMEDIARIOS FINANCIEROS BANCOMEXT. CREDITOS PYMES } \\
\hline BANCO & \multicolumn{1}{c}{ DESTINO } \\
\hline Citibanamex & Capital de Trabajo \\
\hline Banorte & Capital de trabajo y activo fijo. \\
\hline BBVA Bancomer & Capital de trabajo y activo fijo. \\
\hline Santander & $\begin{array}{l}\text { Capital de trabajo y activo fijo. Reestructuraciones o } \\
\text { renovaciones con otros bancos }\end{array}$ \\
\hline Banca Afirme & $\begin{array}{l}\text { Capital de trabajo y activo fijo, para la pre-exportación, } \\
\text { exportación e importación }\end{array}$ \\
\hline Banco BASE & $\begin{array}{l}\text { Capital de trabajo permanente, adquisición de maquinaria y eq. } \\
\text { Eq. de transporte, construcción de bodegas, etc. }\end{array}$ \\
\hline HSBC & Capital de trabajo y activo fijo. \\
\hline BanBajio & Capital de trabajo, activo fijo y proyectos de inversión \\
\hline BX+ & Capital de trabajo, adquisición de activo fijo, excepto terrenos \\
\hline BanRegio & $\begin{array}{l}\text { Capital de trabajo permanente, adquisición de maquinaria y eq, } \\
\text { eq de transporte, construcción de bodegas, etc. }\end{array}$ \\
\hline
\end{tabular}

Fuente: Elaboración propia en base a datos del portal web Bancomext (2017)

\section{IV.VI Uniones de Crédito}

Son organizaciones auxiliares de crédito, constituidas por personas físicas o morales, mismas que son micros y pequeñas empresas y cuyos objetivos son resolver por medio de la cooperación sus necesidades financieras en forma directa y oportuna, así como otros beneficios derivados de actividades realizadas en común por sus socios (abasto, comercialización y asistencia técnica) (Herrera, 1998). 


\section{IV.VII Sociedades Financieras de objeto múltiple}

Las Sociedades Financieras de Objeto Múltiple (SOFOMES) son sociedades anónimas que cuentan con un registro vigente ante la Comisión Nacional para la Protección y Defensa de los Usuarios de Servicios Financieros (CONDUSEF), y cuyo objeto social principal es la realización habitual y profesional de una o más de las actividades de otorgamiento de crédito, arrendamiento financiero o factoraje financiero. (CNBV, 2015).

IV.VIII Financiamiento informal (Villegas \& Ortega, 2002).

Otro medio de financiamiento importante es el informal y a continuación se describen:

Prestamos de parientes o amigo.- Estos créditos casi nunca pagan interés, ya que se basan en la confianza y en la relación cercana.

- Préstamo de negocio.- Este se da en el campo cuando los agricultores o ganaderos reciben algún anticipo de sus clientes. En el caso de los comerciantes, algunos fabricantes les otorgan crédito y ellos, a su vez, para realizar las ventas, otorgan crédito a sus clientes. Una característica para estas transacciones es la buena reputación de la persona.

- El crédito de los aboneros.- Cumple el objetivo de fomentar la actividad económica del que vende y al mismo tiempo, de manera sencilla, da al comprador la posibilidad de adquirir bienes.

- El crédito de los agiotistas.- Estas personas, tienen dinero sobrante para prestar, documentan sus préstamos para garantizarlos, aparte de que solicitan alguna garantía, como joyas, autos o bienes inmuebles. La característica de los agiotistas es que otorgan créditos sin fomentar la actividad productiva o solidaria, simplemente busca el beneficio económico del cobrar un interés muy elevado o quedarse con el bien empeñado.

- Crédito de una caja popular.- otorgan préstamos a sus miembros a menor tasa y sin todos los requisitos que exige un banco.

Muchos empresarios utilizan los créditos personales, tarjetas de crédito, recursos propios o utilidades reinvertidas, para financiarse. 


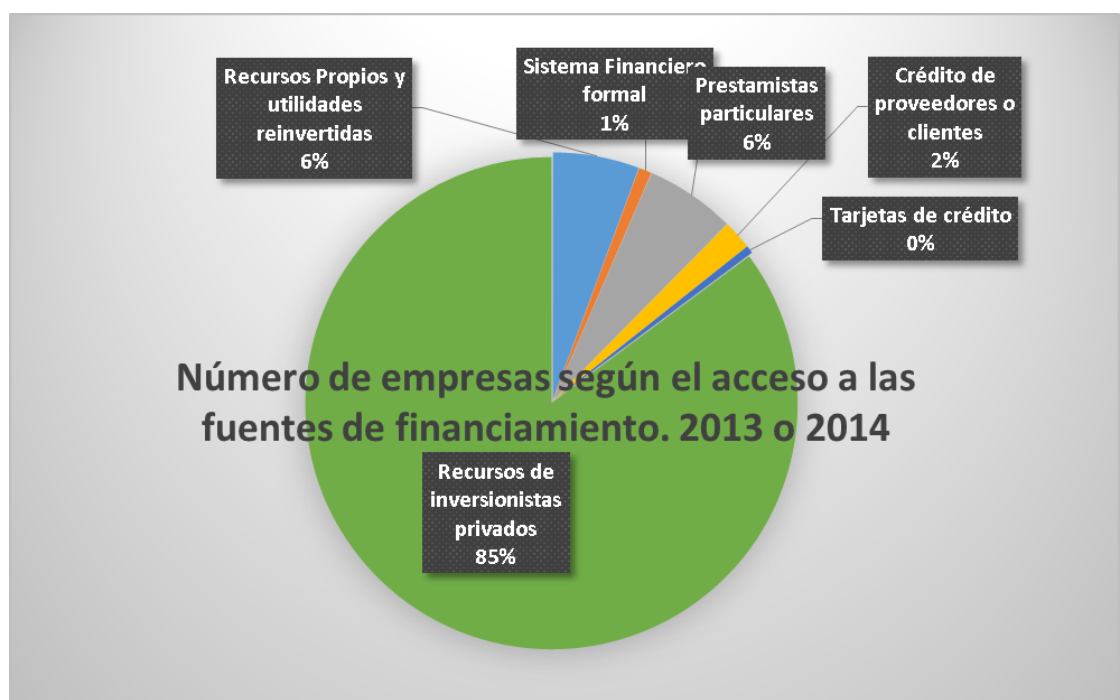

Figura 1. Número de empresas. Elaboración propia con datos del Enaproce 2015 (INEGI, 2016).

En la ENAPROCE 2015 (INEGI, 2016), el 85\% de las MIPYMES dijeron que se financian con recursos de inversionistas privados.

\section{IV.VIX Crowdfunding}

Es un fondeo colectivo. El emprendedor sube su proyecto a una plataforma digital y recibe apoyo de inversionistas, fondeadores y donantes. Hay cuatro tipos: de capital (el inversionista recibe acciones), de deuda (a cambio de una tasa de interés), de donación (el inversionista dona dinero porque le cautivó el proyecto) y de recompensas (el aportador recibe un producto o servicio). (Rodríguez, 2018)

Fue una iniciativa del Fondo Multilateral de Inversiones (FOMIN) del Banco Interamericano de Desarrollo (BID) ejecutado por la Universidad Anáhuac México a través del Centro IDEARSE con la participación de Nacional Financiera, el InstitutoNacional del Emprendedor, la Oficina de Estrategia Digital de la Presidencia de la República, la Comisión Nacional Bancaria y de Valores y la Asociación de Plataformas de Fondeo Colectivo, para impulsar proyectos vía crowdfunding o de financiamiento/fondeo colectivo, ecosistema que está en una fase inicial en México, y que es considerado un mercado alternativo de fondeo para proyectos innovadores a través de plataformas digitales (Crowdfonding México, 2016)

\section{IV.X Apoyo gubernamental}

La Secretaría de Economía creo un órgano administrativo desconcentrado, el Instituto Nacional del Emprendedor (INADEM), el cual finalizo sus actividades en el primer trimestre de 2019 y cuyo objeto era "Instrumentar, ejecutar y coordinar la política nacional de apoyo incluyente a Emprendedores y a las MIPYMES, impulsando su innovación, competitividad y proyección en los mercados nacional e internacional para aumentar su contribución al desarrollo económico y productividad empresarial." 
(INADEM, 2018), cuenta con el Fondo Nacional Emprendedor, el cual financia diversas convocatorias anuales para el apoyo de proyectos y empresas. Antes del INADEM han existido otros programas gubernamentales para el apoyo de las MIPYMES, que han estado a cargo de la Secretaria de Economía.

Los apoyos gubernamentales otorgados por el INADEM, se hacen por medio de convocatorias, tienen la opción de préstamos a fondo perdido, queson aportaciones del gobierno que se hacen a las empresas para impulsar una idea de negocios, pero como ya se mencionó anteriormente este Instituto se creó en el pasado sexenio y el nuevo gobierno planteará otra manera de organización.

En un artículo de la Revista Forbes de México, se menciona que en la política del gobierno del Presidente Andrés Manuel López Obrador (AMLO) el INADEM, no continuará más su labor, ya que se argumenta que ha incurrido en algunas prácticas de corrupción y exceso de burocracia, se dice que ahora las MIPYMES han quedado desamparadas, pues este Instituto también daba asesoría para el acceso al financiamiento y apoyo para el fortalecimiento de las empresas. La actual secretaria de Economía, Graciela Márquez, señaló que se otorgarían 3,000 millones de pesos en microcréditos, para los micronegocios (Mendoza, 2019).

Tabla 5.

Programas federales de promoción y apoyo

\begin{tabular}{ccccc}
\hline \multirow{2}{*}{ Tamaño } & \multicolumn{2}{c}{$\begin{array}{c}\text { Ponocimiento de los } \\
\text { programas }\end{array}$} & \multicolumn{3}{c}{$\begin{array}{c}\text { Durante 2013 y 2014, } \\
\text { solicitaron y recibieron } \\
\text { apoyos }\end{array}$} \\
\cline { 2 - 5 } & Número & $\%$ & Número & $\%$ \\
\hline Micro & 566,799 & $14.3 \%$ & 54,358 & $9.6 \%$ \\
\hline Pequeña & 8,858 & $11.2 \%$ & 910 & $10.3 \%$ \\
\hline Mediana & 1,734 & $10.3 \%$ & 224 & $12.9 \%$ \\
\hline Total & 577,391 & $35.9 \%$ & 55,492 & $32.8 \%$ \\
\hline
\end{tabular}

Fuente: Elaboración propia con datos del Enaproce 2015 (INEGI, 2016)

En la encuesta Enaproce 2015 (INEGI, 2016) el 14.3\% de las MIPYMES tenían conocimiento de los programas federales de promoción y apoyo, y solo el $9.6 \%$ de ellas solicitaron y recibieron dichos apoyos.

La causa principal por la que no solicitaron dichos apoyos es porque el $65 \%$ de las MIPYMES no se habían enterado. 


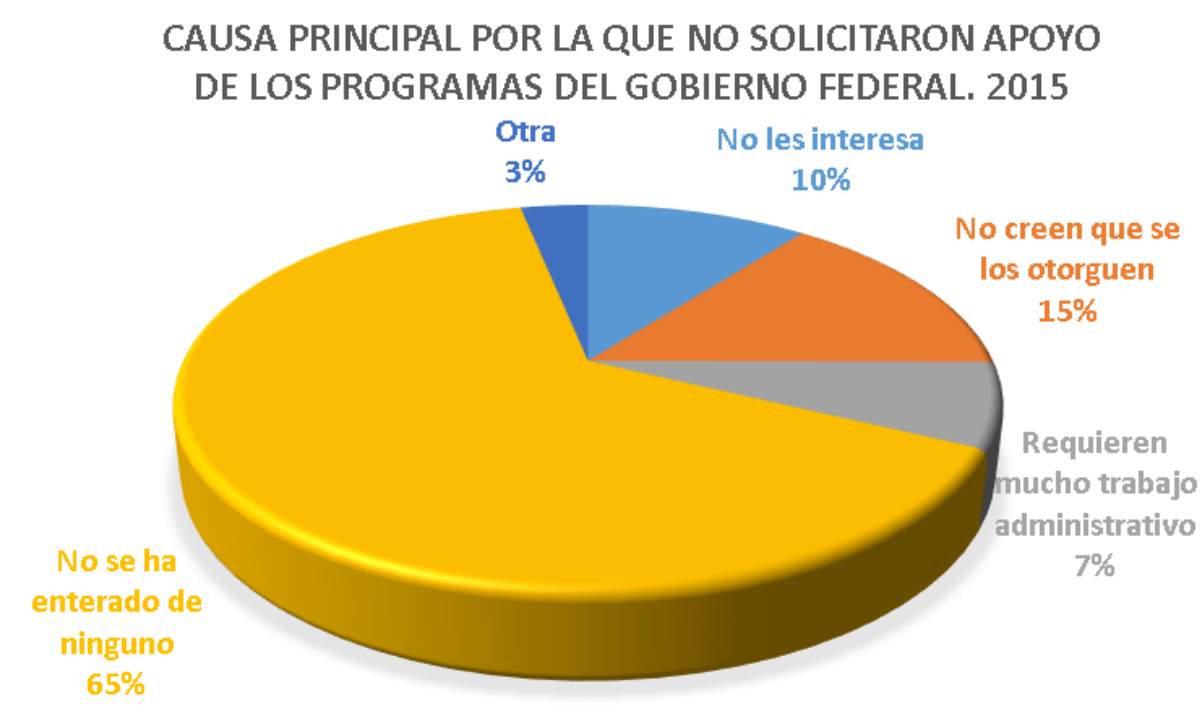

Figura 2. Causa principal. Elaboración propia con datos del Enaproce 2015 (INEGI, 2016)

En la encuesta Enaproce 2015 realizada por el INEGI (2016), se analiza cuáles son los principales problemas que afectan al crecimiento de las empresas, lo que se contesta es: la falta de crédito con un $23 \%$, como principal problema en las microempresas.

Tabla 6

Principales problemas que afectan el crecimiento de las empresas

\begin{tabular}{lrrrrrr}
\hline Tamaño de empresa & \multicolumn{2}{c}{ Micro } & \multicolumn{2}{c}{ Pequeñas } & \multicolumn{2}{c}{ Medianas } \\
\hline Falta de crédito & 895,468 & $23 \%$ & 7,274 & $9 \%$ & 999 & $6 \%$ \\
\hline $\begin{array}{l}\text { Baja demanda de sus productos } \\
\text { (bienes o servicios) }\end{array}$ & 659,758 & $17 \%$ & 9,277 & $12 \%$ & 2,163 & $13 \%$ \\
\hline $\begin{array}{l}\text { Exceso de trámites } \\
\text { gubernamentales e impuestos altos }\end{array}$ & 482,490 & $12 \%$ & 19,395 & $24 \%$ & 4,015 & $24 \%$ \\
\hline Problemas de inseguridad pública & 268,125 & $7 \%$ & 5,948 & $7 \%$ & 884 & $5 \%$ \\
\hline $\begin{array}{l}\text { Competencia de empresas } \\
\text { informales }\end{array}$ & 761,750 & $19 \%$ & 11,853 & $15 \%$ & 2,444 & $15 \%$ \\
\hline No tiene problemas & 499,467 & $13 \%$ & 13,316 & $17 \%$ & 3,372 & $20 \%$ \\
\hline Otros problemas & 385,363 & $10 \%$ & 12,304 & $16 \%$ & 2,876 & $17 \%$ \\
\hline Total empresas & $3,952,422$ & & 79,367 & & 16,754 & \\
\hline \multicolumn{1}{c}{$4,048,543$} & $97.6 \%$ & & $2.0 \%$ & & $0.4 \%$ & \\
\hline
\end{tabular}

Fuente: Elaboración propia con datos del Enaproce 2015 (INEGI, 2016)

De la misma manera la empresa Konfío realizó una encuesta en el año 2018, en donde analiza cuales son los principales retos para las MIPYMES. (Konfío, 2018): 
Tabla 7.

Principales retos para Las PYMES

\begin{tabular}{cccc}
\hline & Micro & Pequeña & Mediana \\
\hline Falta de acceso a crédito & $70.70 \%$ & $68.60 \%$ & $57.10 \%$ \\
\hline Competencia & $8.94 \%$ & $9.73 \%$ & $22.90 \%$ \\
\hline Dificultad para aterrizar planes de negocio & $8.54 \%$ & $8.41 \%$ & $11.49 \%$ \\
\hline Falta de control administrativo & $6.91 \%$ & $8.41 \%$ & $5.71 \%$ \\
\hline Gastos excesivos & $4.88 \%$ & $4.87 \%$ & $2.86 \%$ \\
\hline
\end{tabular}

Fuente: Elaboración propia con datos de Konfío (2018)

Este reporte, también menciona que "los principales atributos que busca un empresario al momento de elegir crédito son: rapidez en el trámite del crédito, pocos requisitos y tasa de interés accesible" (Konfío,2018).

Tabla 8.

Necesidades de crédito

\begin{tabular}{lrrr}
\hline & Micro & Pequeña & Mediana \\
\hline Proceso rápido y sencillo & $54 \%$ & $65 \%$ & $58 \%$ \\
\hline Tasa de interés accesible & $8 \%$ & $8 \%$ & $25 \%$ \\
\hline Confianza con la institución & $23 \%$ & $22 \%$ & $8 \%$ \\
\hline Pocos requisitos & $8 \%$ & $3 \%$ & $8 \%$ \\
\hline
\end{tabular}

Fuente: Elaboración propia con datos de Konfío ( 2018)

\section{IV.XI Tasa de interés}

Por lo que respecta a las tasa de interés promedio cobrada a las PYMES la OCDE en su reporte del año 2018, dice que "en el año 2016, Francia (1.50\%) cobró la tasa de interés más baja a las PYMES, mientras que Chile (9.25\%) cobro el más alto. Por lo que se refiere a México, el reporte señala que esta en 9\%. También dice que a partir de 2013 todos los países de la OCDE han disminuido las tasas de interés (OCDE, 2018).

Otro elemento para tomar en cuenta en el momento de contratar un crédito es el CAT (Costo anual total) que es una medida estandarizada del costo de financiamiento, expresado en términos porcentuales anuales que, para fines informativos y de comparación, incorpora la totalidad de los costos y gastos inherentes a los créditos que otorgan las instituciones. Se calcula con los componentes y metodología establecida por el Banco de México en la Circular 21/2009, y a partir del 17 de noviembre de 2009 el CAT debe calcularse sin considerar el IVA de comisiones ni de intereses (Banxico, 2014)

El siguiente cuadro obtenido del reporte ENAPROCE, 2015, muestra que del total de las MIPYMES solo el $8 \%$ tuvieron acceso a fuentes de financiamiento. También se puede apreciar que la tasa de interés promedio es del $13.86 \%$ 
Tabla 9.

Acceso a fuentes de financiamiento más importante

\begin{tabular}{crcccrc}
\hline \multirow{2}{*}{ Tamaño } & \multicolumn{2}{c}{ Empresas } & \multicolumn{3}{c}{ Número de empresas } \\
\cline { 2 - 7 } & Número & Participación & Estimación total & $\%$ & $\begin{array}{c}\text { No. de } \\
\text { meses }\end{array}$ & $\begin{array}{c}\text { Tasa de } \\
\text { interés }\end{array}$ \\
\hline Micro & $3,952,422$ & $97.6 \%$ & 335460 & $8 \%$ & 16 & $13.86 \%$ \\
\hline Pequeña & 79,367 & $2.0 \%$ & 20458 & $26 \%$ & 24 & $13.13 \%$ \\
\hline Mediana & 16,754 & $0.4 \%$ & 6363 & $38 \%$ & 24 & $11.13 \%$ \\
\hline Total & $4,048,543$ & $100.0 \%$ & 362,282 & & & \\
\hline
\end{tabular}

Fuente: Elaboración propia en base a datos del Enaproce 2015 (INEGI, 2016)

\section{METODOLOGÍA, TÉCNICAS Y MATERIALES EMPLEADOS}

Se visitaron los portales de Internet de las diecisiete principales instituciones bancarias de primer piso, que ofrecen crédito a las PYMES, esta información fue consultada del 12 de marzo al 25 de abril de 2019, algunas de estas instituciones no publican la tasa de interés que cobran.

En cuanto al número de requisitos o documentación solicitada, va de 3 y hasta 12 . Sin embargo se debe considerar que es necesario abrir una cuenta bancaria con la Institución que otorgará el crédito, esto requerirá el pago de comisiones por apertura, por disposición, manejo de cuenta, anualidad, saldo promedio mínimo, entre otros cobros de comisiones, recordando que las comisiones y los intereses causan IVA por lo que se tendrá que pagar un $16 \%$ más.

En cuanto a los intereses que se publican van desde el $10 \%$ y hasta el $118 \%$, algunas tasas son fijas y otras variables (se basan en la TIIE 24-04-19=8.25 Banco de México).

Por lo que la MIPYME tendrá que tomar en cuenta el CAT promedio; que el mínimo es del $14 \%$ y el máximo del $130 \%$ (+ IVA), en donde se están considerando ya todas las comisiones y las tasas de interés que tendrá de pagar.

Tabla 10

Instituciones financieras

Información obtenida del Portal de cada uno de las Instituciones Financieras del 12 de marzo al 25 de abril de 2019

\begin{tabular}{lcc}
\hline \multicolumn{1}{c}{ CONCEPTO } & VALOR & \multicolumn{1}{c}{ OBSERVACIONES } \\
\hline Instituciones Bancarias & 17 & $\begin{array}{l}\text { Banorte, Santander, Afirme, Banregio, Multiva, Scotianbanc, } \\
\text { HSBC, MIFEL, Bancomer, Banamex, Bancobase, Banbajio, BX+, } \\
\text { Unión de Crédito, Inbursa, Banco Azteca, Fundación Dondé }\end{array}$ \\
\hline $\begin{array}{l}\text { Diferentes tipos de } \\
\text { créditos }\end{array}$ & 38 & No todos los créditos presentan sus tasas de interés \\
\hline Línea crédito desde (miles) & 30 & \\
\hline Línea crédito hasta (miles) & 50000 & \\
\hline
\end{tabular}




\begin{tabular}{|c|c|c|}
\hline Plazo mínimo (en meses) & 2 & \\
\hline Plazo máximo (en meses) & 84 & Para la adquisición de un bien inmueble \\
\hline Tasa mínima & $10 \%$ & Hay tasas variables y tasas fijas \\
\hline Tasa máxima & $118 \%$ & $\begin{array}{l}\text { Las tasas variables hacen referencia a la TIIE }=8.25 \% \text { (al } 24 \text { de } \\
\text { abril-2019 de acuerdo al Banco de México) }\end{array}$ \\
\hline CAT promedio mínimo & $14 \%$ & \multirow{2}{*}{ Sin IVA } \\
\hline CAT promedio máximo & $130 \%$ & \\
\hline No de Requisitos mínimos & 3 & \multirow{2}{*}{$\begin{array}{l}\text { Dependerá si es Persona Física o Moral, y si es para un } \\
\text { determinado crédito }\end{array}$} \\
\hline No. de Requisitos máximos & 12 & \\
\hline
\end{tabular}

Fuente: Elaboración propia con datos de portales de internet de diferentes bancos (2019)

Si las tasas de interés presentadas parecen altas, las tasas de interés del financiamiento informal, fluctúan entre $150 \%$ y $250 \%$, según información obtenida de MIPYMES ubicadas en la central de abastos en Cd de Puebla.

Como se puede observar, si hay opciones de crédito con tasas muy altas y cobro de comisiones que incrementan de manera importante el costo total del financiamiento, además de engorrosos trámites administrativos.

\section{V.I Conclusiones}

- Las Microempresas son muy importantes para México, deberían tener una atención integral, no solo darles préstamos a fondo perdido, sino apoyarlas para crecer.

- Toda empresa necesita financiamiento en cualquier momento de su existencia, y el financiamiento en México es caro, con trámites excesivos, así que los empresarios y emprendedores no deben esperar a que alguien les solucione su problema, hay opciones diferentes como el crowdfunding.

- La unión hace la fuerza si las MIPYMES se asocian pueden tener beneficios económicos.

- En la encuesta del ENAPROCE 2015, muchas MIPYMES reportaron estar endeudadas con créditos de inversionistas privados, lo que no se menciona es costo del financiamiento.

- A los Microempresarios les falta cultura financiera, no investigan, no se informan o talvez no necesitan el financiamiento porque ya no quieren crecer.

\section{V.II Propuestas y recomendaciones}

1.- La administración en una MIPYME es un punto clave, para tomarse en cuenta, se mencionan los puntos más importantes (Cualli, 2006):

- El director de la MIPYME no solo debe ser un buen jefe, sino un buen líder, la capacitación puede ayudar.

- La empresa deberá contar con un equilibrio financiero entre ingresos y egresos, las aportaciones de capital son buenas, pero las ventas harán más rentable el negocio, 
por lo tanto "no puede salir nada que no haya entrado".

- Cada peso deberá estar invertido en algo que dará rendimiento a corto plazo.

- La contabilidad y las finanzas deberán estar organizadas, ya que estas generan información, que deberá estar disponible en cualquier momento y que ayudará a la mejor toma de decisiones, es necesario el uso de sistemas informáticos que ayuden a esta tarea.

- El capital humano es muy importante para la empresa, se le debe valorar, motivar, capacitar y hacerlo parte de los objetivos de la empresa.

2.- La formalización de una MIPYME, es un requisito indispensable para cualquier trámite de financiamiento.

3.- Investigar sobre los diferentes tipos de créditos y apoyos que el gobierno otorga, será información muy valiosa.

4.- Analizar para que se requiere el financiamiento:

- Si es para capital de trabajo, el financiamiento deberá ser a corto plazo o la contratación de un crédito revolvente.

- Comprar nuevos activos fijos, el financiamiento deberá ser un crédito simple a largo plazo con la tasa de interés más baja y fija.

- Invertir en un nuevo proyecto, fondearse con apoyos del gobierno, o buscar un crowfonding.

5.- Analizar el flujo efectivo de la empresa tomando en cuenta el pago de capital e intereses que se tendrán que realizar. En los portales de instituciones financieras hay calculadoras financieras que proporcionan el importe mensual a pagar ya con los intereses, algunos bancos lo llaman "Crédito Digital".

6.- Un endeudamiento implica desarrollo y crecimientosi la estrategia está bien determinada, esescalable, adecuada e implementada en el momento preciso.

(Santander, 2019).

7.- Buscar capacitación y asesoría, el líder no lo sabe todo, ni puede hacerlo todo, lo más importante es generar una cultura financiera.

8.- Aprovechar toda la información y educación financiera que ofrece el Banco de México, Nafin, y todos los bancos comerciales en sus portales de internet.

9.- Utilizar la tecnología en favor del financiamiento como: Internet, páginas web, calculadoras financieras, ayuda de los programas de cómputo basado en el software de reconocimiento del lenguaje humano, plataformas de crowdfunding.

10.- Generar una cultura financiera en nuestros niños desde la educación básica, porque 
ellos serán los que formaran las MIPYMES del futuro que tendrán que vencen a muchos gigantes económicos. En los portales de Banxico y NAFIN hay una sección de "Educación económica y financiera" para niños y para los papás de los niños.

\section{REFERENCIAS}

Bancomext. (01 de 01 de 2017). Bancomext Pymes. Recuperado el 05 de 04 de 2019, de Bancomext: https://www.bancomext.com/pymex/productos/

Banxico. (01 de 01 de 2014). Banco de México. (B. d. México, Editor) Recuperado el 21 de 03 de 2019, de Banxicoeduca: http://educa.banxico.org.mx/recursos_banxico_educa/calculadoras/calculadoras-ycomparativos-d.html

CNBV - Comisión Nacional Bancaria y de Valores (30 de 06 de 2015). SOFOMES. Recuperado el 19 de 04 de 2019, de Comisión Nacional Bancaria y de Valores: https://www.cnbv.gob.mx/SECTORES-SUPERVISADOS/OTROSSUPERVISADOS/Descripci\%C3\%B3n-del-Sector/Paginas/SOFOMES-Reguladas.aspx

Crowdfunding México. (01 de 01 de 2016). Asociación de Plataformas de Fondeo Colectivo -AFICO. (AFICO, Editor) Recuperado el 26 de 04 de 2019, de Asociación de Plataformas de Fondeo Colectivo -AFICO: http://www.crowdfundingmexico.mx/investigaciones.html

Cualli. (01 de 01 de 2006). Financiera Cualli. Recuperado el 26 de 04 de 2019, de Financiera Cualli: https://cualli.mx/blog/financiamiento-para-pymes-todo-lo-que-debes-saber/

FIRA. (01 de 01 de 2018). Fideicomisos Instituidos en Relación con la Agricultura. Recuperado el 07 de 04 de 2019, de Fideicomisos Instituidos en Relación con la Agricultura: https://www.fira.gob.mx/Nd/AcercadeNosotros.jsp

Herrera, C. (1998). Fuentes de Financiamiento. México DF: SICCO.

INADEM. (2013 2018). LIBRO BLANCO POLITICA DE MIPYMES/INADEM (2013-2018). CD MEXICO: SECRETARIA DE ECONOMÍA. Recuperado el 21 de 04 de 2019, de https://www.inadem.gob.mx/wp-content/uploads/2018/12/Libro-BlancoPOLI\%CC\%81TICA-DE-MIPYMES.pdf

INEGI. (2016). Estadísticas sobre las Micro, Pequeñas y Medianas empresas del país. Aguascalientes, Ags: INEGI. Recuperado el 06 de 03 de 2018, de http://www.inegi.org.mx/est/contenidos/proyectos/encuestas/establecimientos/otras/en aproce/default_t.aspx

Konfío (31 de 12 de 2018). Konfío.mx. Recuperado el 20 de 04 de 2019, de Konfío.mx: https://konfio.mx/reporte-credito-pyme

Mendoza E, V. (15 de 02 de 2019). 30 Promesas 2019 | El emprendimiento después del Inadem. Forbes de México. Recuperado el 21 de 04 de 2019, de https://www.forbes.com.mx/30- 
promesas-2019-el-emprendimiento-despues-del-inadem/

Nafin. (01 de 01 de 2008). Nacional Financiera. Recuperado el 05 de 04 de 2019, de Nacional Financiera: https://www.nafin.com/portalnf/content/home/home.html

OCDE. (01 de 01 de 2018). OECD SMEs, Regions, Cities. Recuperado el 21 de 04 de 2019, de OECD SMEs, Regions, Cities: www.oecd.org/cfe/sme

RAE. (01 de 01 de 2019). Diccionario de la lengua española. (RAE, Editor) Recuperado el 21 de 03 de 2019, de Diccionario de la lengua española: http://dle.rae.es/?id=EsuT8Fg

Rodríguez C, V., Bao G., R., \& Cárdenas L., L. (2014). Formulación y Evaluación de Proyectos. México DF: Limusa.

Rodríguez, G. (06 de 12 de 2018). Líder empresarial. Recuperado el 26 de 04 de 2019, de Líder empresarial: https://www.liderempresarial.com/como-puedo-financiar-mi-startup-sin-recurrir-ala-banca/

Santander. (19 de 02 de 2019). Santander. Recuperado el 20 de 04 de 2019, de Santander: https://www.santanderpyme.com.mx/detalle-noticia/endeudarte-si-pero-inteligentemente.html

Secretaría de Economía. (15 de 03 de 2018). Secretaría de Economía. Recuperado el 19 de 04 de 2019, de http://www.2006-2012.economia.gob.mx/mexicoemprende/empresas/microempresario

Villegas H., E., \& Ortega O., R. (2002). Sistema Financiero de México. Cd de México: McGraw Hill.

\section{Glosario}

BANCOMEXT.- Banco Nacional de Comercio Exterior.

CAT.- Costo Anual Total

ENAPROCE.- Encuesta Nacional sobre Productividad y Competitividad de las Micro, Pequeñas y Medianas Empresas

FIRA.- Fideicomisos Instituidos en Relación con la Agricultura

INADEM.- Instituto Nacional del Emprendedor

INEGI.- Instituto Nacional de Estadística y geografía

MIPYMES.- Micro pequeñas y medianas empresas

NAFIN.- Nacional Financiera

OCDE.- Organización para la Cooperación y el Desarrollo Económico

PYMES.- Pequeñas y Medianas Empresas

SOFOM.-Sociedad Financiera de Objeto Múltiple 